\title{
Kindler syndrome protein Kindlin-1 is mainly expressed in adult tissues originating from ectoderm/endoderm
}

\author{
ZHAN Jun ${ }^{1,2}$, YANG Mei ${ }^{1,2}$, ZHANG Jing ${ }^{1,2}$, GUO YongQing ${ }^{3}$, LIU Wei ${ }^{4}$ \\ \& ZHANG HongQuan ${ }^{1,2^{*}}$ \\ ${ }^{1}$ Key Laboratory of Carcinogenesis and Translational Research, Ministry of Education, State Key Laboratory of Natural and Biomimetic \\ Drugs, Peking University Health Science Center, Beijing 100191, China; \\ ${ }^{2}$ Laboratory of Molecular Cell Biology and Tumor Biology, Department of Anatomy, Histology and Embryology, Peking University Health \\ Science Center, Beijing 100191, China; \\ ${ }^{3}$ Department of Thoracic Surgery, Sino-Japan Friendship Hospital, Beijing 100100, China; \\ ${ }^{4}$ Department of Immunology, Third Military Medical University, Chongqing 400038, China
}

Received July 21, 2014; accepted October 3, 2014; published online January 14, 2015

\begin{abstract}
Mutations of integrin-interacting protein Kindlin-1 cause Kindler syndrome and deregulation of Kindlin-1 is implicated in human cancers. The Kindlin-1-related diseases are confined in limited tissue types. However, Kindlin-1 tissue distribution and the dogma that governs Kindlin-1 expression in normal human body are elusive. This study examined Kindlin-1 expression in normal human adult organs, human and mouse embryonic organs by immunohistochemical analyses. We identified a general principle that the level of Kindlin-1 expression in tissues is tightly correlated with the corresponding germ layers from which these tissues originate. We compared the expression of Kindlin-1 with Kindlin-2 and found that Kindlin-1 is highly expressed in epithelial tissues derived from ectoderm and endoderm, whereas Kindlin-2 is mainly expressed in mesoderm-derived tissues. Likewise, Kindlin-1 was also found highly expressed in endoderm/ectoderm-derived tissues in human and mouse embryos. Our findings indicate that Kindlin-1 may play an importance role in the development of endoderm/ectoderm related tissues.
\end{abstract}

Kindlin-1, epithelial tissue, ectoderm, endoderm, embryo

Citation: Zhan J, Yang M, Zhang J, Guo YQ, Liu W, Zhang HQ. Kindler syndrome protein Kindlin-1 is mainly expressed in adult tissues originating from ectoderm/endoderm. Sci China Life Sci, 2015, 58: 432-441, doi: 10.1007/s11427-014-4775-2

Kindlin-1 (encoded by the FERMT1 gene) belongs to a family of evolutionary conserved proteins. Kindlin-1 was found to locate at cell-matrix adhesion sites and to bind to the cytoplasmic tail of integrin $\beta$ subunit and increase its affinity for ligands [1-3]. Mutations in FERMT-1 gene cause an autosomal-recessive disease, Kindler syndrome (KS) with skin disorder featured by skin blistering, poikiloderma, photosensitivity and carcinogenesis [4]. Mechanically, Kindlin- 1 forms molecular complexes with $\beta$ integrin, $\alpha$-actinin, migfilin, and focal adhesion kinase and regulates cell shape and migration. Kindlin-1 controls cell motility by

*Corresponding author (email: Hongquan.Zhang@bjmu.edu.cn) activation of Rho family GTPasesRac1, RhoA and Cdc42, leading to the phosphorylation of their downstream effectors p21-activated kinase 1, LIM kinase, and cofilin [5]. KS also displays other clinical symptoms including chronic erosive gingivitis, dysphagia, esophageal and urethral strictures, ectropion, and lethal neonatal intestinal epithelial dysfunction and the enhanced risk of muco-cutaneous squamous cell carcinoma [1,5-7]. All these symptoms are caused by Kindlin-1 loss in the corresponding organs. However, the Kindlin-1 expression profile in the whole human body remains unknown. In comparison, we previously found that the Kindlin family protein Kindlin-2 is 
highly expressed in mesoderm-derived tissues [8]. Despite sequence homology between Kindlin-1 and Kindlin-2, some of their biological functions are different and they cannot functionally compensate to each other [9-11]. Therefore, one may ask if Kindlin-1 and Kindlin-2 are differentially expressed in the human body. Apart from KS, Kindlin-1 was also proved to play an important role in tumorigenesis. It has been reported that Kindlin-1, as a regulator of TGF $\beta$ signaling, plays an important role in breast cancer growth and lung metastasis [12]. Kindlin-1 enhances migration and invasion of pancreatic [13] and colon cancer cells [14]. The inhibitory role of Kindlin-1 was reported in lung [11] and skin cancers [15]. Mechanistically, Kindlin-1 controls keratinocyte adhesion through $\beta 1$-integrins and proliferation and differentiation of cutaneous epithelial stem cells by promoting $\alpha v \beta 6$ integrin-mediated transforming growth factor- $\beta$ (TGF- $\beta$ ) activation and inhibiting Wnt- $\beta$-catenin signaling through integrin-independent regulation of Wnt ligand expression [15]. The mechanism of inhibiting tumorigenesis is opposite to the effect of Kindlin-2 [11]. Kindlin-1 was also found to enhance axon growth on inhibitory chondroitin sulfate proteoglycans and promote sensory axon regeneration [16].

The tissue distribution of the Kindlin-1 genes has been analyzed by multiple tissue in situ hybridization in mice. The results showed that Kindlin-1 is preferentially expressed in epithelia [17]. However, nothing is known about the localization of Kindlin-1 protein in embryo in situ. The scenario behind this embryonic origin of these organs with Kindlin-1 expression remains mysterious.

In order to answer the above questions, we first examined Kindlin-1 protein expression in normal adult human organs. The characteristic distribution of Kindlin-1 in epithelia is related to tissue germinal origination, driving us to examine Kindlin-1 expression in human and mouse embryonic organs. Meanwhile, we compared Kindlin-1 expression profile with that of Kindlin-2.

\section{Materials and methods}

\subsection{Normal and tumor tissues}

We obtained samples of cancer tissues and normal control tissue from the patients who underwent tumor resection at Sino-Japan Friendship Hospital between July 2010 and September 2013. Five aborted fetuses (from 20 to 2 weeks) samples were collected from Department of Obstetrics and Gynecology, Sino-Japanese Friendship Hospital between July 2007 and September 2013 (Permit Number: ZRLW-5 ZRLW-7).

\subsection{Animal experiment}

Ten-week-old ICR pregnant female mice were bred in a room with $12 \mathrm{~h} / 12 \mathrm{~h}$ light-dark cycles and given food and water ad libitum. We acquired the embryos of mice $16.5 \mathrm{~d}$ after copulation and the whole embryos were formalin-fixed and paraffin-embedded (Permit Number: LA2011-73).

\subsection{Immunohistochemistry (IHC)}

All slides were formalin-fixed and paraffin-embedded. Deparaffinization and hydration were performed and followed by abolishing endogenous peroxidase activity using $0.3 \%$ hydrogen peroxide for $30 \mathrm{~min}$ and microwave for antigen retrieval in $10 \mathrm{mmol} \mathrm{L}^{-1}$ sodium citrate buffer $(\mathrm{pH} \mathrm{6.0)}$ for $20 \mathrm{~min}$. We used affinity-purified polyclonal anti-Kindlin-1 (PKU Animal Facility) antibody at $2 \mu \mathrm{g} \mathrm{mL} \mathrm{m}^{-1}$ and anti-Kindlin-2 mouse monoclonal antibody (1:1000 dilution; Millipore, USA) $2 \mu \mathrm{g} \mathrm{mL}^{-1}$ to perform these experiments. The primary antibody was used at $4^{\circ} \mathrm{C}$ overnight. Then PV9000 2-step plus Poly-HRP Anti-mouse/rabbit IgG Detection System (Zhong Shan Jin Qiao, China) was applied. The streptavidin-biotin-peroxidase method was used for detection and diaminobenzidine was applied for substrate (ChemMate Detection Kit, DAKO, Denmark) [19]. Hematoxylin was used for counterstaining. Negative controls were performed by omitting the use of primary antibody.

\section{Results}

\subsection{Kindlin-1 was highly expressed in ectoderm/ endoderm-derived tissues of human adults}

Kindlin-1 was highly expressed in tongue stratified squamous epithelium (Figure 1A, black arrows) and taste bud (Figure 1A, red arrow). In esophageal stratified squamous epithelium (Figure 1B), Kindlin-1 was expressed differently in all three layers. In the basal layer (Figure 1B, black arrow), Kindlin-1 was mainly located in nuclei; in the middle layer (Figure 1B, red arrow), Kindlin-1 was moderately expressed in the cytoplasm. In the surface layer (Figure 1B, black arrow head), Kindlin-1 became more highly expressed again. Kindlin-1 was found highly expressed in the epithelial of gastric gland (Figure 1C), especially at the bottom of the gland (Figure 1C, black arrows). A regular pattern in gastrointestinal expression was found, in which Kindlin-1 was high in the simple columnar epithelium of duodenum (Figure 1D, black arrows), jejunum (Figure 1E, black arrows) and colon (Figure 1F, black arrows), but negative in the mesenchyme of villi (Figure 1D, red arrows; Figure 1E, red arrows). Kindlin-1 was found expressed neither in the germinal center (Figure 1F, red arrow) nor in the lymphoid nodule cap of gastrointestinal tract (Figure 1F, black arrow head). Kindlin-1 was found widely expressed in the liver including liver cells and bile ducts (Figure 1G, black arrow) but negative in the blood vessel (Figure 1G, red arrow). In the pancreas, the islet (Figure 1H, black arrow) and epithe- 

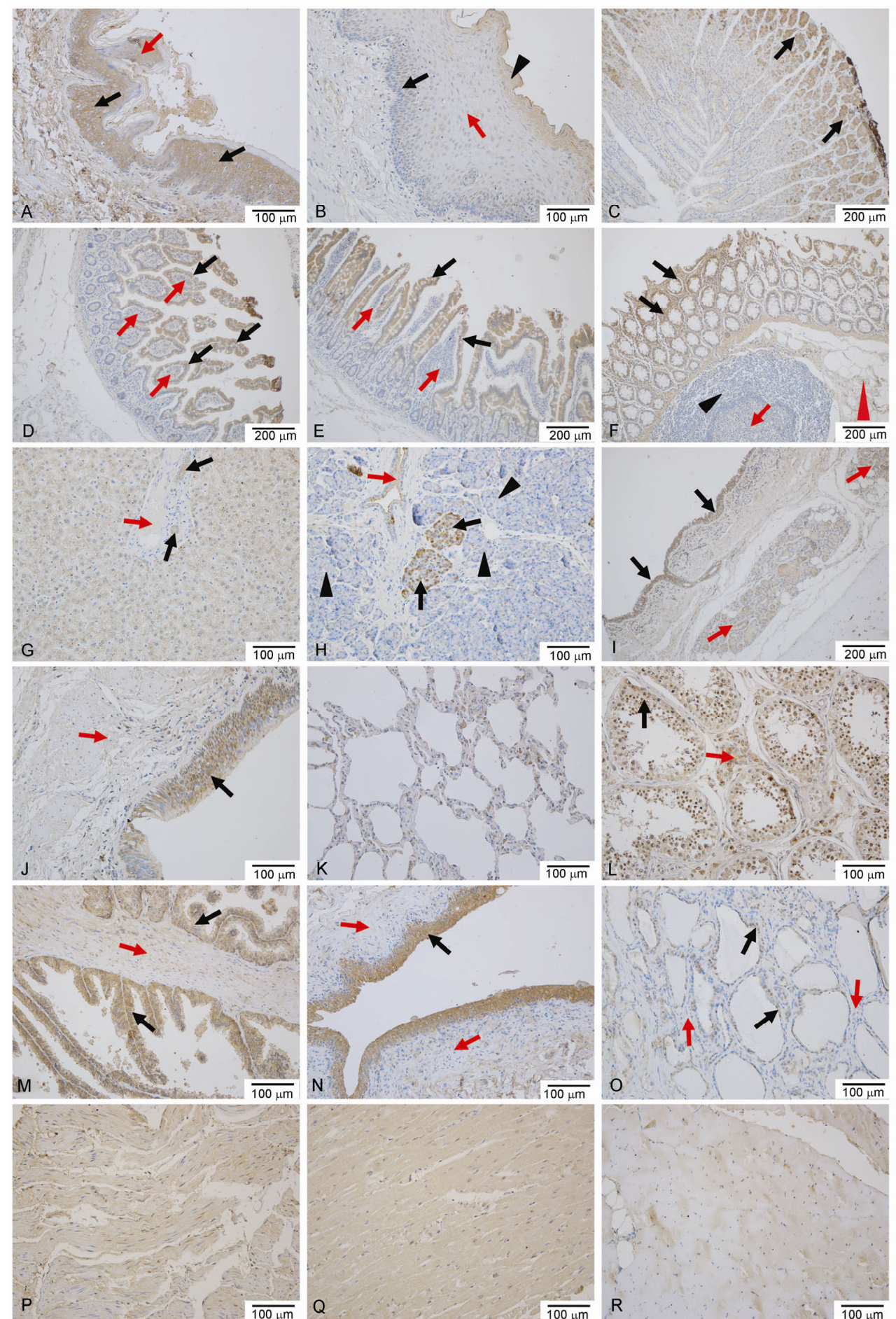

Figure 1 Expression of Kindlin-1 in tissues of human adult. A, Tongue. Black arrows: stratified squamous epithelium; red arrow: taste bud. B, Esophageal stratified squamous epithelium. Black arrow: basal layer; red arrow: middle layer; black arrow head: surface layer. C, Fundic gland of gastric. Black arrows: simple columnar epithelium of gastric gland. D, Duodenum. Black arrows: simple columnar epithelium of duodenum; red arrows: interstitial substance. E, Jejunum. Black arrows: simple columnar epithelium; red arrows: mesenchyme. F, Colon. Black arrows: simple columnar epithelium; red arrow: germinal center lymphoid nodule in colon; black arrow head: cap of lymphoid nodule in colon; red arrow head: mesenchyme. G, Liver. Black arrows: interlobular bile duct in hepatic portal area; red arrow: interlobular vein in hepatic portal area. H, Pancreas. Black arrows: islet; red arrow: interlobular duct; black arrow head: alveoli. I, Trachea. Black arrows: pseudo-stratified ciliated columnar epithelium tissue; red arrows: tracheal gland. J, Trachea. Black arrow: pseudo-stratified ciliated columnar epithelium; red arrow: interstitial substance. K, Lung. L, Testis. Black arrow: seminaferous epithelium; red arrow: interstitial cells of Leydig. M, Prostate. Black arrows: epithelium of prostate; red arrow: mesenchyme. N, Bladder. Black arrow: transitional epithelium of bladder; red arrow: mesenchyme. O, Thyroid. Black arrows: simple cubic epithelium; red arrows: mesenchyme. P, Smooth muscle. Q, Cardiac muscle tissues in heart. R, Skeletal muscles. 
lium of the duct (Figure 1H, red arrow) expressed a high level of Kindlin-1. However, the alveoli (Figure 1H, black arrow heads) expressed a low level of Kindlin-1. In the respiratory system, high Kindlin-1 expression was detected in pseudo-stratified ciliated columnar epithelium (Figure 1I and J, black arrows) and glands (Figure 1I, red arrows) of trachea but not in the connective tissue of trachea and the alveoli of the lung (Figure 1K). In the testis, positive signal can be detected both in the seminiferous epithelium (Figure 1L, black arrow) and in stromal cells (Figure 1L, red arrow). The epithelium of the prostate (Figure 1M, black arrows), transitional epithelium of the bladder (Figure 1N, black arrow) and simple cubic epithelium of the thyroid (Figure 1O, black arrows) all expressed extremely high levels of Kindlin-1 with negative expression in connective tissues (Figure 1M-O, red arrows). In smooth muscle (Figure 1P), cardiac muscle (Figure 1Q) and skeletal muscle tissues (Figure 1R), Kindlin-1was found low or not expressed. Taken together, Kindlin-1 is highly expressed in ectoderm/endoderm-derived tissues including epithelia from the tongue, esophagus, stomach, intestine and respiratory track, but low or moderately expressed in mesoderm-derived tissues including smooth muscle, cardiac muscle and skeletal muscle tissues.

\subsection{Kindlin-1 was highly expressed in ectoderm/ endoderm-derived tissues of human embryos}

In the human embryonic esophagus, Kindlin-1 was highly expressed in the stratified squamous epithelium (Figure 2A, black arrow) and low or moderately expressed in the connective tissue (Figure 2A, red arrow). In the epithelium of human embryonic stomach (Figure 2B, black arrows), duodenum (Figure 2C, black arrows), jejunum (Figure 2D, black arrows) and colon (Figure 2E, black arrows), Kindlin1 was strongly expressed at the bottom of glands in particular (Figure 2D and E, red arrows). The human embryo liver displayed a similar trend of Kindlin-1 expression as the human adult liver. Kindlin-1 was expressed in the hepatocyte and bile duct (Figure 2F, black arrows), but showed no signal in the blood vessel (Figure 2F, red arrow). Distinct from human adult pancreas (Figure $1 \mathrm{H}$ ), in addition to the islet (Figure 2G, red arrow), Kindlin-1 was found highly expressed in the embryonic alveoli of the pancreas (Figure $2 \mathrm{G}$, black arrows), suggesting that Kindlin-1 may play an important role in the embryonic alveoli development. The epithelia of respiratory track (Figure $2 \mathrm{H}$, black arrows) and alveoli (Figure 2H, red arrow; Figure 2I, black arrows) showed positive Kindlin-1 expression in human embryos. In the urogenital system of human embryos, positive signals of Kindlin-1 were detected in different regions of renal tubules (Figure 2J and $\mathrm{K}$ ), transitional epithelium of the bladder (Figure 2L) and germ cells (Figure 2M and N, black arrows). In particular, the distal convoluted tubules (Figure 2J and K, black arrow heads) expressed a higher level of Kindlin-1 than proximal convoluted tubules (Figure $2 \mathrm{~J}$ and $\mathrm{K}$, red arrows). Interestingly, Kindlin-1 expression was negative in the glomerulus (Figure 2J and $\mathrm{K}$, black arrows) that is mainly composed of blood vessels. Primary oocytes (Figure $2 \mathrm{M}$, red arrows) surrounded by follicular cells (Figure 2M, black arrows), seminiferous epithelium (Figure 2N, black arrows) and testicular interstitial cells (Figure 2N, red arrows) all displayed high levels of Kindlin-1 expression. In glandular epithelium of pituitary (Figure 2O, black arrows), spinal cord neurons (Figure 2P, red arrow), nerve fibers (Figure 2P, black arrow), and peripheral myelinated nerve fibers (Figure 2Q, black arrows), Kindlin-1 was all positively stained. Skin dysfunction is the main clinical symptoms caused by Kindlin-1 deficiency. Accordingly, we found that skin is one of the organs that give the highest expression of Kindlin-1. Kindlin-1 displayed strong positive signals in stratified squamous epithelium of embryonic skin (Figure2R, black arrows) and the epithelium of hair follicles (Figure 2R, red arrows). However, in dermis of the skin, Kindlin-1 is negative (Figure2R, black arrow heads).

\subsection{Kindlin-1 was weakly expressed in meso- derm-derived tissues of human embryos}

Human embryonic thymus (Figure 3A) showed no Kindlin1 expression. In cardiac-vesicular system including largesized arteries (Figure 3B), medium-sized arteries (Figure 3C) and the heart (Figure 3D), Kindlin-1 was demonstrated to be weakly expressed. Kindlin-1 showed no expression in smooth muscles of the bladder (Figure 3E), a representative of smooth muscle tissues of the whole embryo. Hyaline is another example of mesoderm-derived tissue in which Kindlin-1 expression was very low (Figure 3F).

\subsection{Kindlin-1 was highly expressed in ectoderm/endo- derm-derived but not mesoderm-derived tissues of mouse embryos}

As what we observed in human embryos, Kindlin-1 was also highly expressed in the mouse embryonic skin (Figure $4 \mathrm{~A}$, black arrow). In the middle region of the mouse embryo (Figure 4B), Kindlin-1 was located at the edge of alveoli of the lung (Figure 4B and C, black arrows), at the alveoli and ductal epithelia of pancreas (Figure 4B, red arrows; Figure 4D, black arrows), at the epithelium of intestine (Figure 4B, black arrow head; Figure 4E, black arrows), and at the liver (Figure 4F). In neurons of the spinal cord, Kindlin-1 expression was positive (Figure 4G, black arrows). However, Kindlin-1 expression was not detectable in mouse embryonic cardiac-vesicular wall (Figure $4 \mathrm{H}$, black arrow) and bone tissues (Figure 4I, black arrows). Collectively, the Kindlin-1 expression profile in mouse embryos was similar to that in human embryos. 


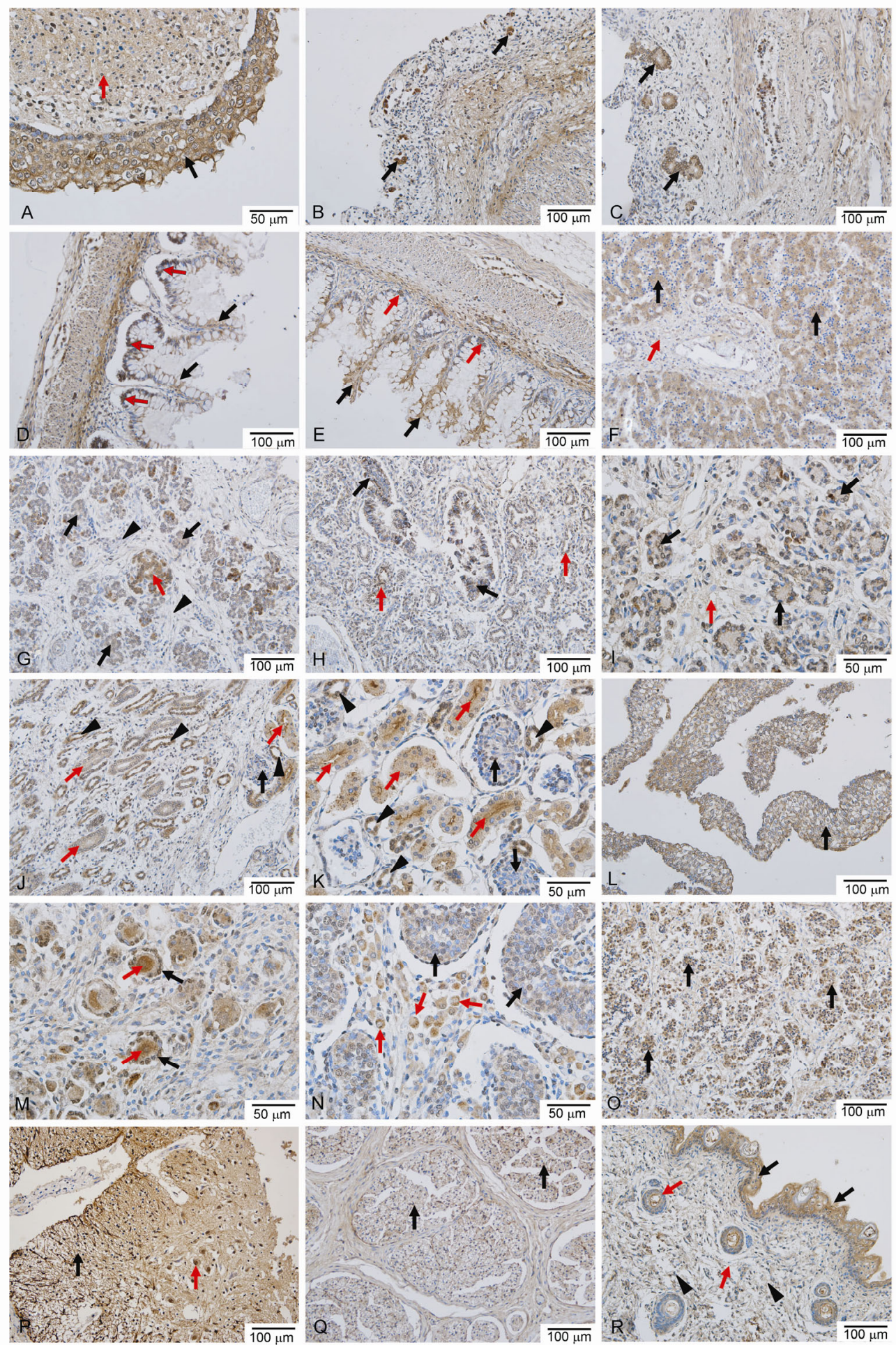

Figure 2 Kindlin-1 is highly expressed in ectoderm/endoderm-derived tissues of human embryo. A, Esophagus. Black arrow: stratified squamous epithelium; red arrow: connective tissue. B, Stomach. Black arrows: epithelium. C, Duodenum. Black arrows: epithelium; red arrow: interstitial substance. D, Jejunum. Black arrows: epithelium; red arrows: bottom of the gland. E, Colon. Black arrows: epithelium; red arrows: bottom of the gland. F, Liver. Black arrows: hepatocyte; red arrow: hepatic portal area. G, Pancreas. Black arrows: alveoli; red arrow: islet; black arrow head: mesenchyme. H, Lung. Black arrows: epithelium tissue of trachea; red arrows: epithelium of pulmonary alveolus. I, Lung. Black arrows: pulmonary alveolus; red arrow: mesenchyme. J, Medulla of kidney. Black arrow: glomerular; red arrows: proximal convoluted tubule; black arrow heads: distal convoluted tubule. K, Cotex of kidney. Black arrows: glomerular; red arrows: proximal convoluted tubule; black arrow heads: distal convoluted tubule. L, Transitional epithelium of bladder. M, Ovary. Black arrows: follicle cells; red arrows: primary oocytes. N, Testis. Black arrows: seminaferous epithelium; red arrows: interstitial cells of Leydig. O, Pituitary. Black arrows: glandular epithelium of pituitary. P, Spinal cord. Black arrow: nerve fibers; red arrow: neuron. Q, Sciatic nerve. Black arrow: peripheral myelinated nerve fibers; red arrow: perineurium. R, Skin. Black arrows: stratified squamous epithelium; red arrows: hair follicle. 

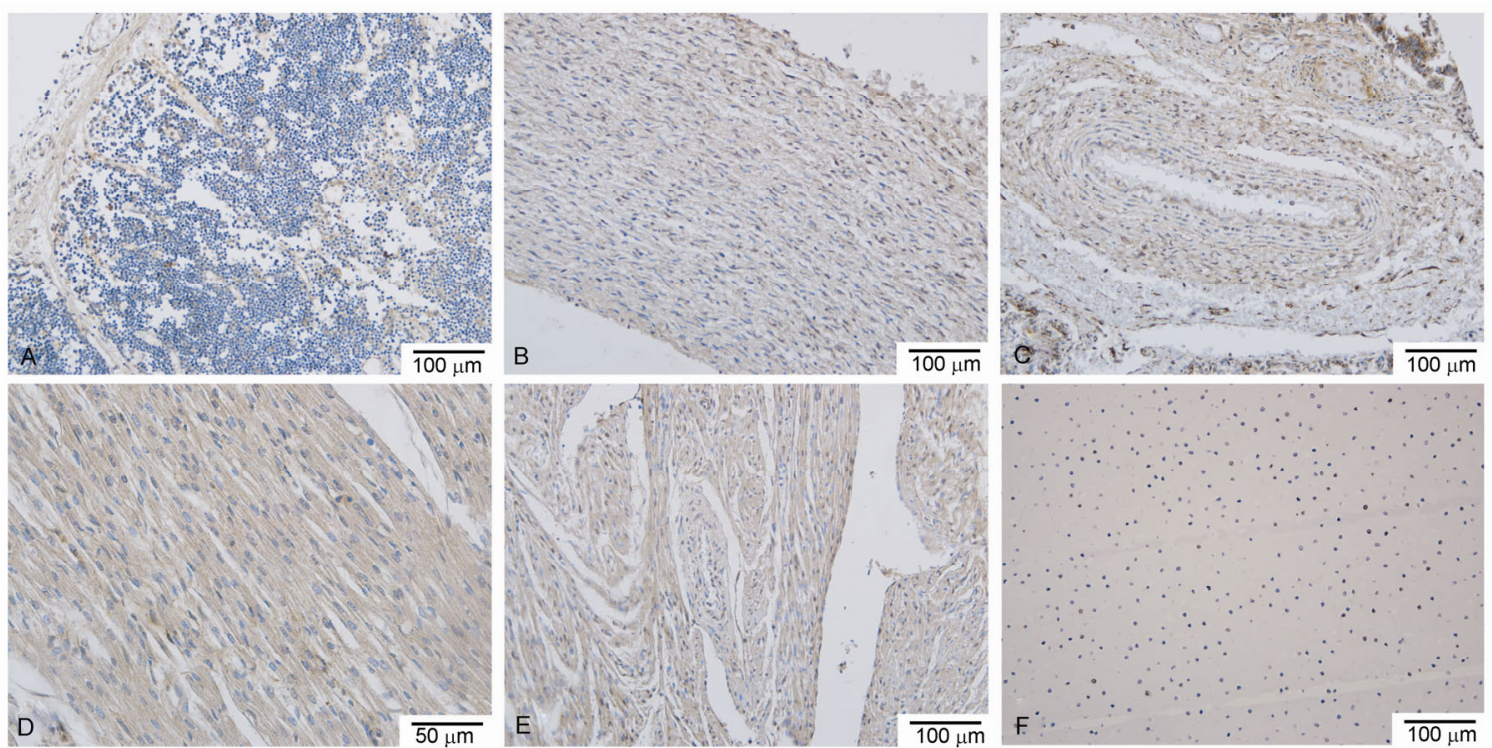

Figure 3 Kindlin-1 is negatively or weakly expressed in mesoderm-derived tissues of human embryo. A, Thymus. B, Large-sized artery. C, Medium-sized artery. D, Cardiac muscles. E, Smooth muscles in the wall of bladder. F, Hyaline.
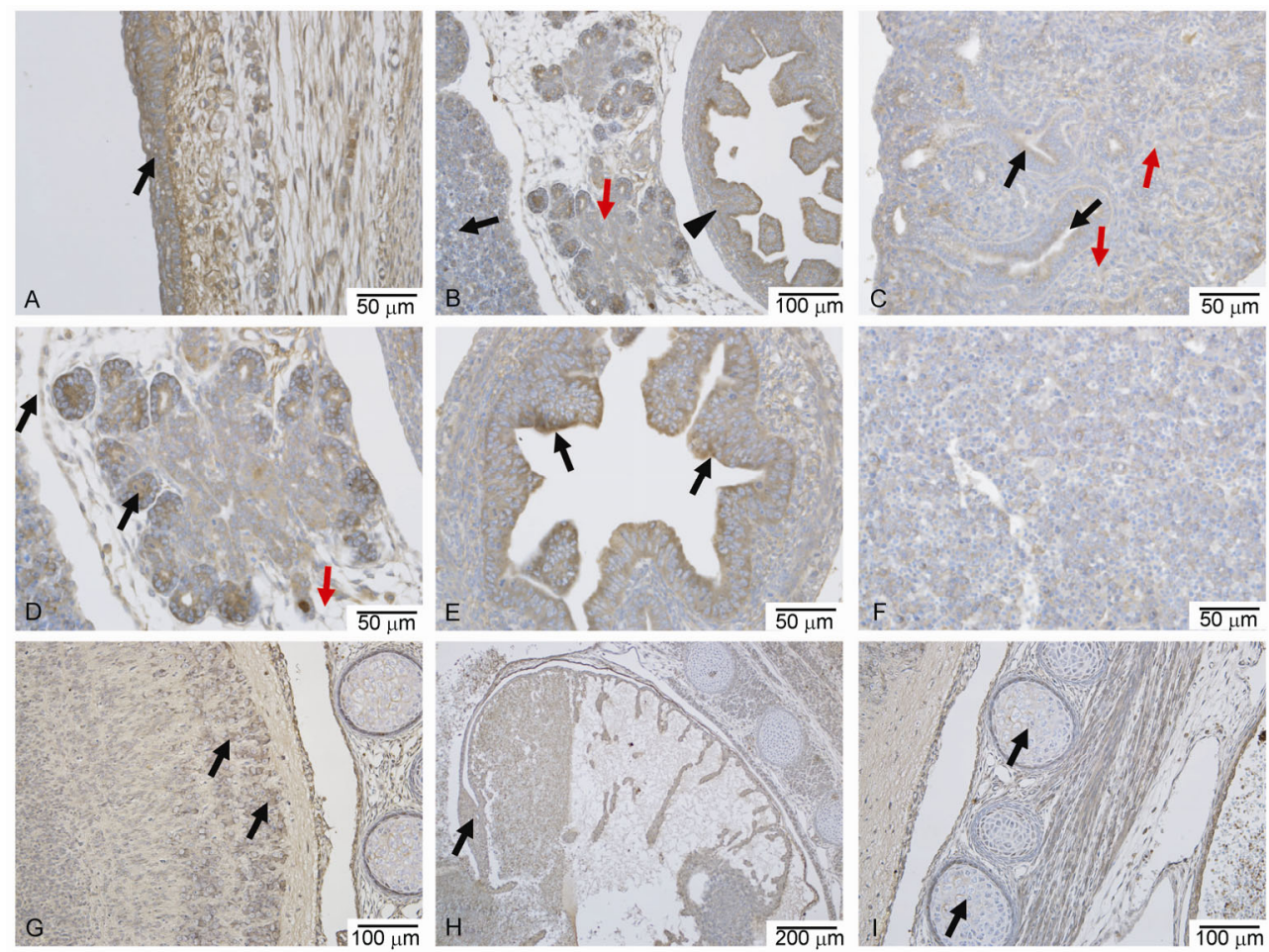

Figure 4 Kindlin-1 expression pattern in day 16.5 mouse embryo. A, Skin. Black arrow: stratified squamous epithelium. B, Abdomen of mouse embryo. Black arrow: lung; red arrow: pancreas; black arrow head: intestine. C, Lung. Black arrows: epithelium tissue of trachea; red arrows: mesenchyme. D, Pancreas. Black arrows: pancreas alveoli; red arrow: mesenchyme. E, Intestines. Black arrows: mouse embryonic intestines epithelial tissues. F, Liver. G, Spinal cord. Black arrows: neuron. H, Heart. Black arrow: wall of the heart. I, Vertebral column. Black arrows: bone tissue.

\subsection{Kindlin-1 and Kindlin-2 were conversely expressed in human tissues}

To identify the difference and relationship between Kindlin-1 and Kindlin-2 in human tissues, Kindlin-1 and
Kindlin-2 expressions were compared in the same human embryonic tissues. For the representative tissues, Kindlin-1 was highly expressed in the epithelium of the intestine (Figure 5A, black arrow), alveoli (Figure 5C, black arrows) and duct (Figure 5C, red arrows) of pancreas, epithelium of 

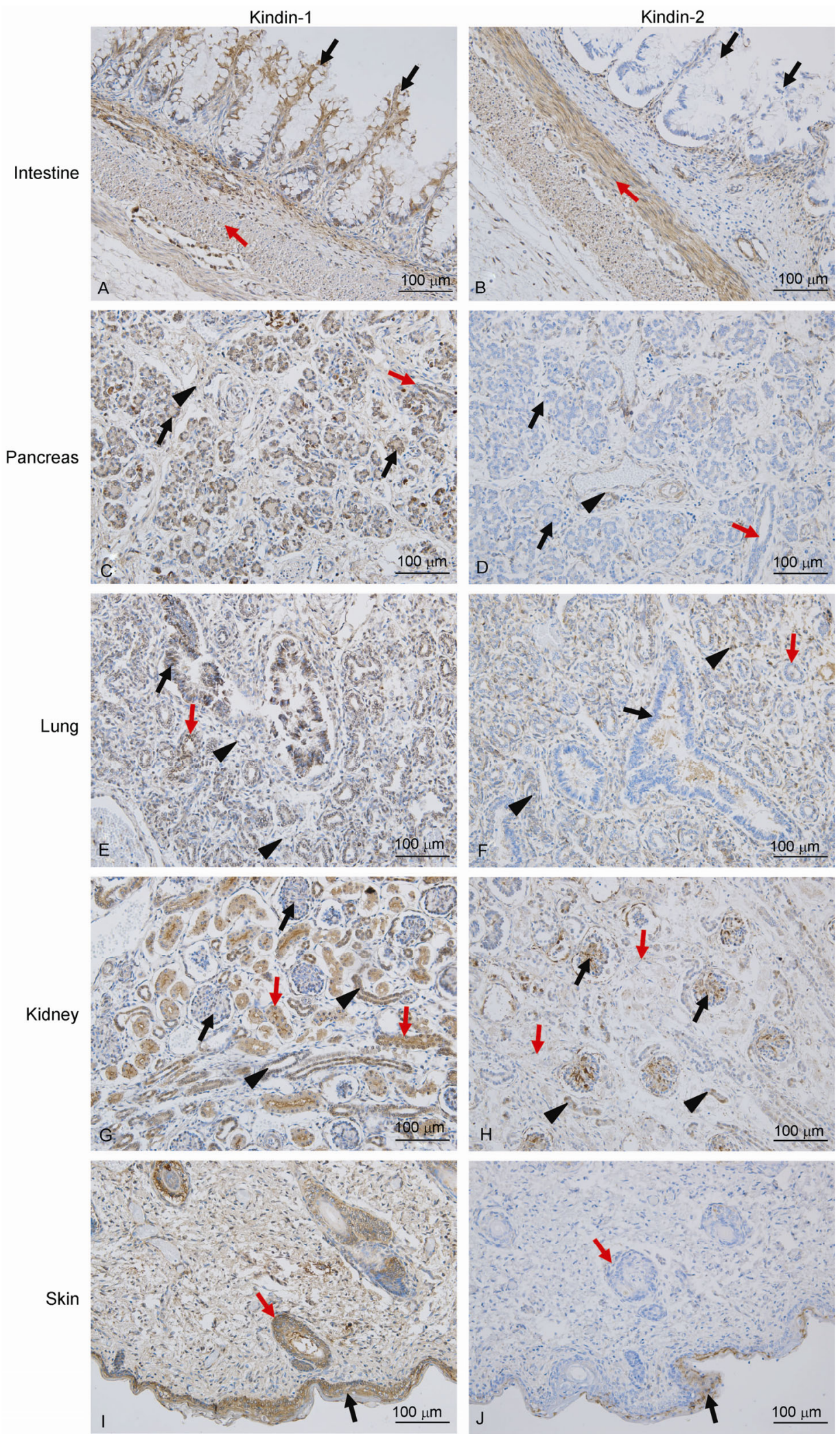

Figure 5 Comparison of Kindlin-1 and Kindlin-2 expression in human embryonic tissues. Left panel is Kindlin-1 expression in human embryonic tissues. Right panel is Kindlin-2 expression in human embryonic tissues. A and B, Intestine. Black arrows: epithelium; red arrow: smooth muscle. C and D, Pancreas. Black arrows: pancreatic alveoli; red arrow: duct; black arrow head: mesenchyme. E and F, Lung. Black arrow: epithelium tissue of trachea; red arrow: epithelium of pulmonary alveolus. $\mathrm{G}$ and $\mathrm{H}$, Kidney. Black arrows: glomerular; red arrows: proximal convoluted tubule; black arrow heads: distal convoluted tubule. I and J, Skin. Black arrow: stratified squamous epithelium; red arrow: hair follicle. 
the trachea (Figure 5E, black arrow), lung (Figure 5E, red arrow) and renal tubes (Figure 5G, red arrow and black arrow head); in contrast, Kindlin-2 displayed no or weak expression (Figure 5B, D, F and $\mathrm{H}$ ) in corresponding tissues where Kindlin-1 had high expression. However, for the representative tissues where Kindlin-1 showed no or low expression including those of smooth muscles in the intestine wall (Figure 5A, red arrow), the connective tissues in the pancreas (Figure 5C, black arrowhead), lung (Figure 5E) and glomerulus (Figure 5G, black arrows), Kindlin-2 was highly expressed at the corresponding positions (Figure 5B, $\mathrm{D}, \mathrm{F}$ and $\mathrm{H}$ ). As a general tendency, Kindlin-1 was expressed in epithelial tissues that are derived from the ectoderm/endoderm, whereas Kindlin-2 was mainly expressed in connective tissues, including vesicles and muscles originating from the mesoderm. However, Kindlin-1 and Kindlin-2 were both highly expressed in the stratified squamous epithelium of the skin, which is an exception (Figure 5I and J).

\section{Discussion}

In this investigation, first, we found that Kindlin-1 was highly expressed in epithelial tissues derived from digestive, respiratory, urogenital, and endocrine systems as well as the skin. Second, we found that the spinal cord and nerve fibers expressed high levels of Kindlin-1 as well. Third, the muscle, cardiac-vesicular, bone and all other connective tissues showed low or no Kindlin-1 expression. Interestingly, all the epithelial and nerve tissues are derived from the ectoderm/endoderm. The Kindlin-1 expression profile remains identical in both human adults and embryos, suggesting that Kindlin-1 expression in tissues is highly associated with their germ disk origins. Deficiency and mutation of FERMT1 causes autosomal recessive skin disorder, the KS [19], indicating the importance of Kindlin-1 in the formation and development of ectoderm/endoderm-derived tissues and organs. The finding that Kindlin- 1 is widely expressed in the tissues derived from the ectoderm/ endoderm suggests that $\mathrm{KS}$ is the dysfunction of tissues derived from the ectoderm/endoderm. In addition to skin epithelial cell fragility and atrophy that are commonly seen in $\mathrm{KS}$, we anticipate that loss of normal Kindlin-1 functions in tissues or organs derived from the ectoderm/endoderm may lead to other clinical symptoms including chronic erosive gingivitis, dysphagia, esophageal and urethral strictures, ectropion, and an increased risk of mucosa-cutaneous squamous cell carcinoma. The relationship between Kindlin-1 expression in various tissues and their embryonic origins is summarized in Table 1.

In this study, we found for the first time that islet of the pancreas expressed a high level of Kindlin-1. Kindlin-1 was also highly expressed in endocrine cells, indicating that Kindlin-1 may play a role in endocrine function and related diseases which warrant further study. We previously reported that Kindlin-2 was highly expressed in mesoderm originated organs but weakly expressed in ectoderm/ endoderm originated organs [8]. In this study, we found that Kindlin-1 expression in human tissues is contrary to that of Kindlin-2. In the same organs of human embryo, Kindlin-1 is highly expressed at cells or tissues where Kindlin-2 is negative; whereas Kindlin-1 is negative at cells or tissues where Kindlin-2 is strongly expressed. This contrary relationship between Kindlin-1 and Kindlin-2 prevails in most organs. In our previous study on the opposite role of Kindlin-1 and Kindlin-2 in lung cancers [11], we hypothesized that Kindlin-1 and Kindlin-2 are differentially expressed in various tissues and regulate the status of epithelia and mesenchyme. Recent reports support our hypothesis. First, it was reported that Kindlin-1 controls keratinocyte adhesion through $\beta 1$-class integrins and proliferation and differentiation of cutaneous epithelial stem cells by promoting $\alpha v \beta 6$ integrin-mediated TGF- $\beta$ activation and inhibiting Wnt- $\beta$-catenin signaling through integrin-independent regulation of Wnt ligand expression [15]. Kindlin-1 plays a previously unknown and essential task of controlling cutaneous epithelial stem cell homeostasis by balancing TGF- $\beta$ mediated growth-inhibitory signals and Wnt- $\beta$-cateninmediated growth-promoting signals [15]. Second, Kindlin-1 deficient cells will lose epithelial phenotype [20]. Third, Kindlin-2 promotes tumor invasion and metastasis in most of cancer tissues including non-small-cell lung cancer [11], breast cancer [21], bladder cancer [22], and gastric cancer [23]. Fourth, through activating Ras and the downstream ERK1/2 and Akt signaling pathways, Kindlin-2 plays an important role in regulating renal tubular epithelial to mesenchymal transition [24,25]. Kindlin-2 physically interacts with both T $\beta$ RI and Smad3, promoting the activation of TGF- $\beta /$ Smad signaling and contributing to the pathogenesis of tubulointerstitial fibrosis [26]. All these reports support that Kindlin-1 promotes differentiation and mesenchymal to epithelial transition, while Kindlin-2 promotes dedifferentiation and epithelial to mesenchymal transition.

In addition, there is an exception of our dogma that some tissues or cells express both Kindlin-1 and Kindlin-2, such as the stratified squamous epithelium of the skin and the stromal cells of testis. But another report uncovered functional differences between Kindlin-1 and Kindlin-2 in keratinocytes. They found that both Kindlin-1 and Kindlin-2 localize to focal adhesions in wild-type cells, only Kindlin-1localizes to integrin- $\beta 6$-rich adhesions of integrin- $\beta 1$-null cells. Despite the presence of Kindlin-1, knockdown of Kindlin-2 in wild-type keratinocytes impaired cell spreading. Their data reveal consequences of differences in the association of two homologous Kindlin isoforms and suggest that despite their similarities, different Kindlins are likely to have unique functions [27]. In addition, Kindlin-1 and Kindlin-2 were found to have overlapping roles in maintaining epithelial integrity and the phenotype of 
Table 1 Comparison of Kindlin-2 expression among organs derived from different embryonic dermal layers ${ }^{\text {a) }}$

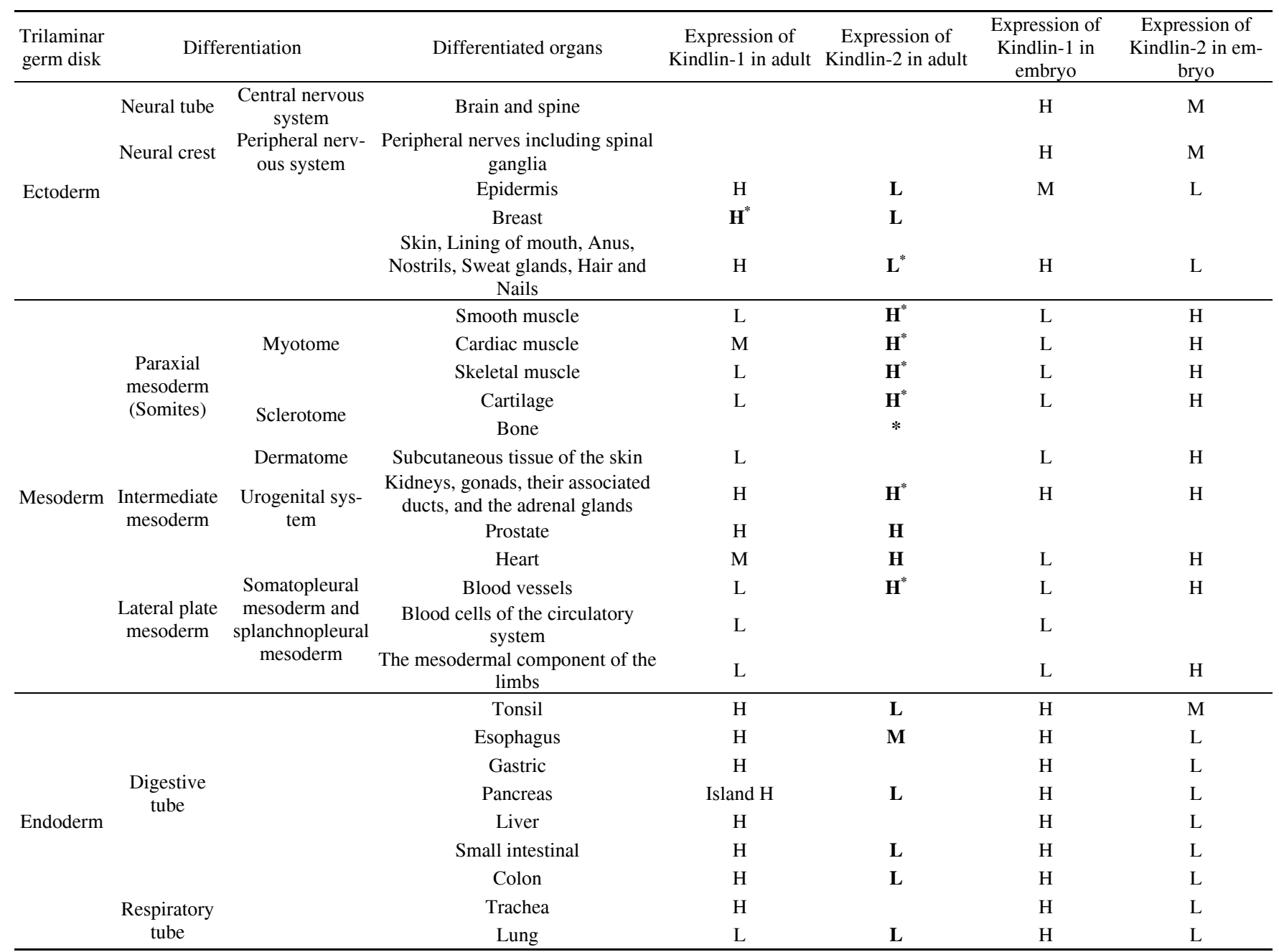

a) $\mathrm{H}$, high expression; $\mathrm{L}$, low expression. *, Being reported previously to play important roles in this organ. Bold $\mathrm{H}$ and $\mathrm{L}$ indicate results from other studies.

Kindlin-1-deficient cells can be modulated by regulating Kindlin-2 gene expression and vice versa [10]. Kindlin-1 and Kindlin-2 can functionally compensate for each other, at least in part [10]. All these findings suggest that despite unique functions of Kindlin-1 and Kindlin-2, there is cooperation between the two in the body.

In summary, we found that integrin-interacting protein Kindlin-1, contrary to Kindlin-2 in expression pattern, is highly expressed in ectoderm/endoderm-derived tissues in both human adults and embryos. Our findings suggest that Kindlin-1 may play an important role in the development of ectoderm/endoderm-derived organs. This study is helpful for understanding the role of Kindlins in normal tissue homeostasis and cancer susceptibility.

\section{The authors declare that they have no conflict of interest.}

The Ethic Committee of Peking University Health Science Center has approved the current study for mouse experiments (Permit Number:
LA2011-73). The Ethic Committee of Sino-Japan Friendship Hospital has approved the current study using lung cancer, breast cancer, pancreas cancer, adrenal cancer, prostate cancer, and relative normal tissues (Permit Number: ZRLW-7). The procedures for handling mice and human materials were in accordance with the ethical standard of the Helsinki Declaration of 1975 (as revised in 2008) concerning Human and Animal Rights, and they followed out policy concerning Informed Consent as shown on Springer.com.

This work was supported by the National Natural Science Foundation of China (81301802, 81230051, 30830048, 31170711), Grant of Ministry of Science and Technology of China (2013CB910501, 2010CB912203, 2010CB529402), Program for Introducing Talents of Discipline to Universities (111 Project) of Ministry of Education, Beijing Natural Science Foundation (7120002), Peking University grants (BMU20120314, BMU20130364, BMU20130373), and a Leading Academic Discipline Project of Beijing Education Bureau.

1 Ussar S, Moser M, Widmaier M, Rognoni E, Harrer C, GenzelBoroviczeny O, Fässler R. Loss of Kindlin-1 causes skin atrophy and lethal neonatal intestinal epithelial dysfunction. PLoS Genet, 2008, 4: e1000289 
2 Montanez E, Ussar S, Schifferer M, Bosl M, Zent R, Moser M, Fässler R. Kindlin-2 controls bidirectional signaling of integrins. Genes Dev, 2008, 22: 1325-1330

3 Moser M, Nieswandt B, Ussar S, Pozgajova M, Fässler R. Kindlin-3 is essential for integrin activation and platelet aggregation. Nat Med, 2008, 14: 325-330

4 Kern JS, Herz C, Haan E, Moore D, Nottelmann S, von Lilien T, Greiner P, Schmitt-Graeff A, Opitz OG, Bruckner-Tuderman L, Has C. Chronic colitis due to an epithelial barrier defect: the role of kindlin-1 isoforms. J Pathol, 2007, 213: 462-470

5 Has C, Bruckner-Tuderman L. Molecular and diagnostic aspects of genetic skin fragility. J Dermatol Sci, 2006, 44: 129-144

6 Lai-Cheong JE, Tanaka A, Hawche G, Emanuel P, Maari C, Taskesen M, Akdeniz S, Liu L, McGrath JA. Kindler syndrome: a focal adhesion genodermatosis. British J Dermatol, 2009, 160: 233-242

7 Sadler E, Klausegger A, Muss W, Deinsberger U, Pohla-Gubo G, Laimer M, Lanschuetzer C, Bauer JW, Hintner H. Novel KIND1 gene mutation in Kindler syndrome with severe gastrointestinal tract involvement. Arch Dermatol, 2006, 142: 1619-1624

8 Zhan J, Yang M, Chi X, Zhang J, Pei X, Ren C, Guo Y, Liu W, Zhang H. Kindlin-2 expression in adult tissues correlates with their embryonic origins. Sci China Life Sci, 2014, 57: 690-697

9 Lai-Cheong JE, Ussar S, Arita K, Hart IR, McGrath JA. Colocalization of kindlin-1, kindlin-2, and migfilin at keratinocyte focal adhesion and relevance to the pathophysiology of Kindler syndrome. $\mathrm{J}$ Invest Dermatol, 2008,128: 2156-2165

$10 \mathrm{He}$ Y, Esser P, Heinemann A, Bruckner-Tuderman L, Has C. Kindlin-1 and -2 have overlapping functions in epithelial cells implications for phenotype modification. Am J Pathol, 2011, 178: 975-982

11 Zhan J, Zhu X, Guo Y, Wang Y, Qiang G, Niu M, Hu J, Du J, Li Z, Cui J, Ma B, Fang W, Zhang H. Opposite role of Kindlin-1 and Kindlin-2 in lung cancers. PLoS One, 2012, 7: e50313

12 Sin S, Bonin F, Petit V, Meseure D, Lallemand F, Bieche I, Bellahcène A, Castronovo V, de Wever O, Gespach C, Lidereau R, Driouch K. Role of the focal adhesion protein kindlin-1 in breast cancer growth and lung metastasis. J Natl Cancer Inst, 2011, 103: 1323-1337

13 Mahawithitwong P, Ohuchida K, Ikenaga N, Fujita H, Zhao M, Kozono S, Shindo K, Ohtsuka T, Aishima S, Mizumoto K, Tanaka M Kindlin-1 expression is involved in migration and invasion of pancreatic cancer. Int J Oncol, 2013, 42: 1360-1366

14 Weinstein EJ, Bourner M, Head R, Zakeri H, Bauer C, Mazzarella R. URP1: a member of a novel family of PH and FERM domain-containing membrane-associated proteins is significantly over-expressed in lung and colon carcinomas. Biochim Biophys Acta, 2003, 1637: 207-216
15 Rognoni E, Widmaier M, Jakobson M, Ruppert R, Ussar S, Katsougkri D, Böttcher RT, Lai-Cheong JE, Rifkin DB, McGrath JA, Fässler R. Kindlin-1 controls Wnt and TGF-beta availability to regulate cutaneous stem cell proliferation. Nat Med, 2014, 20: 350-359

16 Tan CL, Andrews MR, Kwok JC, Heintz TG, Gumy LF, Fassler R, Fawcett JW. Kindlin-1 enhances axon growth on inhibitory chondroitin sulfate proteoglycans and promotes sensory axon regeneration. J Neurosci, 2012, 32: 7325-7335

17 Ussar S, Wang HV, Linder S, Fassler R, Moser M. The Kindlins: subcellular localization and expression during murine development. Exp Cell Res, 2006, 312: 3142-3151

18 Fuchs-Telem D, Nousbeck J, Singer A, McGrath JA, Sarig O, Sprecher E. New intragenic and promoter region deletion mutations in FERMT1 underscore genetic homogeneity in Kindler syndrome. Clin Exp Dermatol, 2014, 39: 361-367

19 Yu J P, Ma Q, Zhang B, Ma R, Xu X, Li M, Xu W, Li M. Clinical application of specific antibody against glypican-3 for hepatocellular carcinoma diagnosis. Sci China Life Sci, 2013, 56: 234-239

20 Qu H, Wen T, Pesch M, Aumailley M. Partial loss of epithelial phenotype in kindlin-1-deficient keratinocytes. Am J Pathol, 2012, 180: 1581-1592

21 Yu Y, Wu J, Guan L, Qi L, Tang Y, Ma B, Zhan J, Wang Y, Fang W, Zhang H. Kindlin 2 promotes breast cancer invasion via epigenetic silencing of the microRNA200 gene family. Int J Cancer, 2013, 133: 1368-1379

22 Talaat S, Somji S, Toni C, Garrett SH, Zhou XD, Sens MA, Sens DA. Kindlin-2 expression in arsenite- and cadmium-transformed bladder cancer cell lines and in archival specimens of human bladder cancer. Urology, 2011, 77: 1507.e1-7

23 Shen Z, Ye Y, Kauttu T, Seppanen H, Vainionpaa S, Wang S, Mustonen H, Puolakkainen P. Novel focal adhesion protein kindlin-2 promotes the invasion of gastric cancer cells through phosphorylation of integrin beta1 and beta3. J Surg Oncol, 2013, 108: 106-112

24 Hirschberg R. Kindlin-2: a new player in renal fibrogenesis. J Am Soc Nephrol, 2013, 24: 1339-1340

25 Wei X, Wang X, Xia Y, Tang Y, Li F, Fang W, Zhang H. Kindlin-2 regulates renal tubular cell plasticity by activation of Ras and its downstream signaling. Am J Physiol Renal Physiol, 2014, 306: F271-278

26 Wei X, Xia Y, Li F, Tang Y, Nie J, Liu Y, Zhou Z, Zhang H, Hou FF. Kindlin-2 mediates activation of TGF-beta/Smad signaling and renal fibrosis. J Am Soc Nephrol, 2013, 24: 1387-1398

27 Bandyopadhyay A, Rothschild G, Kim S, Calderwood DA, Raghavan S. Functional differences between kindlin-1 and kindlin-2 in keratinocytes. J Cell Sci, 2012, 125: 2172-2184

Open Access This article is distributed under the terms of the Creative Commons Attribution License which permits any use, distribution, and reproduction in any medium, provided the original author(s) and source are credited. 